SCMT4

Las Vegas, USA, August 7-11, 2016

\title{
Incorporating Depth-Temperature Profile in Presence of AC Cross- Anisotropy to Enhance Accuracy of Pavement Stress-Strain
}

\author{
Rafiqul A. Tarefder ${ }^{1 \mathrm{a}}$, and Mesbah U. Ahmed ${ }^{1 \mathrm{~b}}$

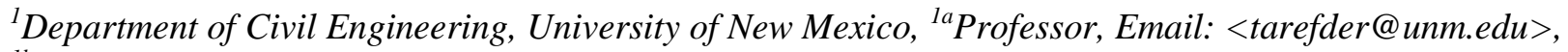 \\ ${ }^{1 b}$ Gradaute Research Assistant, Email: <mahmed@unm.edu>.
}

\begin{abstract}
Accuracy of stress-strain during a pavement analysis can be significantly compromised due to ignorance of non-homogenous distribution of Asphalt Concrete (AC) modulus which results from its temperaturedependency and temperature variation over the depth. To address this issue, a study is performed to investigate whether accuracy of the pavement responses, i.e., stress-strain, can be enhanced by incorporating temperature variation over the depth of an AC layer. A dynamic Finite Element Model (FEM) of an instrumented pavement section on MP 141, Interstate-40 (I-40) is developed to determine the pavement responses. Two different material models are developed: temperature-dependent and crossanisotropic viscoelastic model for AC layer and nonlinear elastic and stress-dependent model for unbound layers. The FEM simulated pavement deflections are compared to field deflections under a Falling Weight Deflectometer (FWD) test load after incorporating the material models. Once the FEM is validated, pavement deflections, stress, and strains are determined incorporating both differential and average temperature constant over the depth of an AC layer. It is observed that pavement responses are not highly affected due to non-homogenous distribution of $\mathrm{AC}$ modulus which results from the temperature variation in AC layer. Therefore, incorporation of depth-temperature profile will not enhance the accuracy of pavement stress-strain, and thereby, pavement distress analysis.
\end{abstract}

\section{INTRODUCTION}

It is a common practice to assign average Asphalt Concrete (AC) temperature which is assumed constant over the depth of an AC layer during a pavement analysis to determine stress-strain (Wang and Al-Qadi 2013). In reality, AC temperature is not constant or uniformly distributed and it may lead to a nonhomogenous distribution of AC modulus over the depth of an AC layer (Diefenderfer 2002, and Herb et al. 2006). There is a possibility that the accuracy of pavement analysis is compromised due to ignorance of non-homogenous distribution of temperature-dependent AC modulus.

In addition to non-homogenous modulus distribution, the $\mathrm{AC}$ shows cross-anisotropy, i.e., $\mathrm{AC}$ moduli along horizontal and vertical directions are not equal. In a study by Ahmed et al. (2015), it is observed that the AC cross-anisotropy significantly affects pavement stress-strain. Incorporation of AC cross-anisotropy leads to high amount of tensile strain at the bottom of the AC layer and vertical strain pavement layers. These strains are affected more by the cross-anisotropy at high temperature. Nonlinear elastic and stressdependency of unbound layer is another important material property. Recently, Tarefder et al. (2016) 
observed that the pavement responses due to AC cross-anisotropic variation are enhance whenever the unbound layer nonlinearity is incorporate to a pavement model.

To this end, it is necessary to investigate whether the incorporation of differential temperature over the depth in an AC layer affects the pavement deflection, stress, and strain in presence of both AC crossanisotropy and unbound layer nonlinearity. Incorporation of depth-temperature will be strongly recommended if the difference between the pavement responses considering differential and average constant temperature over the depth is considerably high.

\section{Objective}

Main goal of this study is to investigate whether pavement responses, such as deflection, stress, and strain, are significantly affected due to incorporation of differential temperature distribution over AC depth. Specific objectives are as follows:

- Develop a dynamic FEM incorporating temperature-dependent and cross-anisotropic viscoelastic model for AC as well as nonlinear elastic and stress-dependent model for unbound layers.

- Determine the pavement deflections, stress, and strain incorporating the two earlier mentioned depthtemperature distributions.

\section{Development of FEM}

\section{Model Geometry}

Figure 1 shows the geometry of the FEM, which is developed based on an instrumented pavement section at mile post 141 (MP 141) on Interstate 40 (I-40). It consists of four major layers: AC at the surface, a mix of 50\% granular aggregate and 50\% Recycled Asphalt Pavement (RAP) material at the base, Process-Place and Compacted (PPC) layer at the subbase, and a subgrade soil layer. The AC layer consists of three lifts each with a thickness of 3.5 in. $(88.9 \mathrm{~mm})$. The subbase or PPC layer is prepared by processing existing base and/or subgrade materials and then, compacting it in place. The thickness of the base is 6 in. (152.4 $\mathrm{mm}$ ) and the subbase is 8 in. (203.2 mm). Figure 1 shows that horizontal asphalt strain gauges (HASGs), vertical asphalt strain gauges (VASGs), and Earth pressure cells (EPC) were installed in this pavement section.

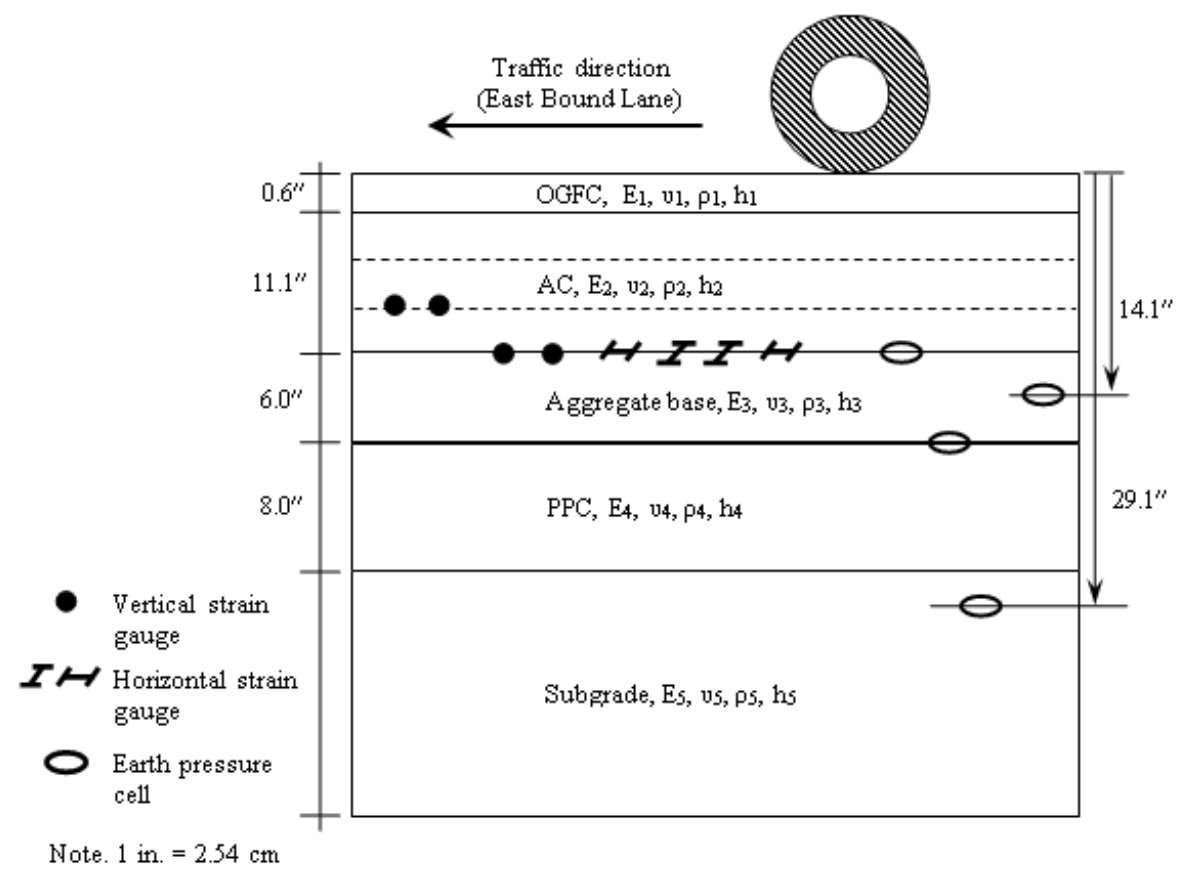

Figure 1. Instrumented pavement section on MP141, Rio Puerco, New Mexico 
The quarter cube geometry was selected to develop the model (see Figure 2). The depth and horizontal length of a model were selected to diminish the effect of stress near the boundary according to Duncan et al. (1968). In this study, the depth of the model was taken 50 times the loading radius and horizontal length was taken more than 12 times the loading radius. Wave reflection by the boundary is one of the major concerns in a dynamic analysis, which may occur due to the insufficient distance to the boundary (Petyt 1990). Therefore, the final dimensions, i.e., length, width, and depth, of this entire model were selected to be 300 in. $x 300$ in. $x 300$ in. $(7.62 \mathrm{~m}$ x $7.62 \mathrm{~m}$ x $7.62 \mathrm{~m})$. The numbers of layers as well as thicknesses of every layer were assigned according to the instrumented section described earlier.

\section{Mesh and Boundary}

An 8-noded brick element (C3D8) was used for the mesh generation (see Figure 2). It is a common practice to assign fine mesh near the loading region to capture the stress gradient and coarser mesh further from that region. Length of the smallest element is $0.6 \mathrm{in} .(15 \mathrm{~mm})$ based on the mesh sensitivity analysis. An edge biased structure meshing pattern was used to obtain a smooth transition from fine mesh to coarse mesh.

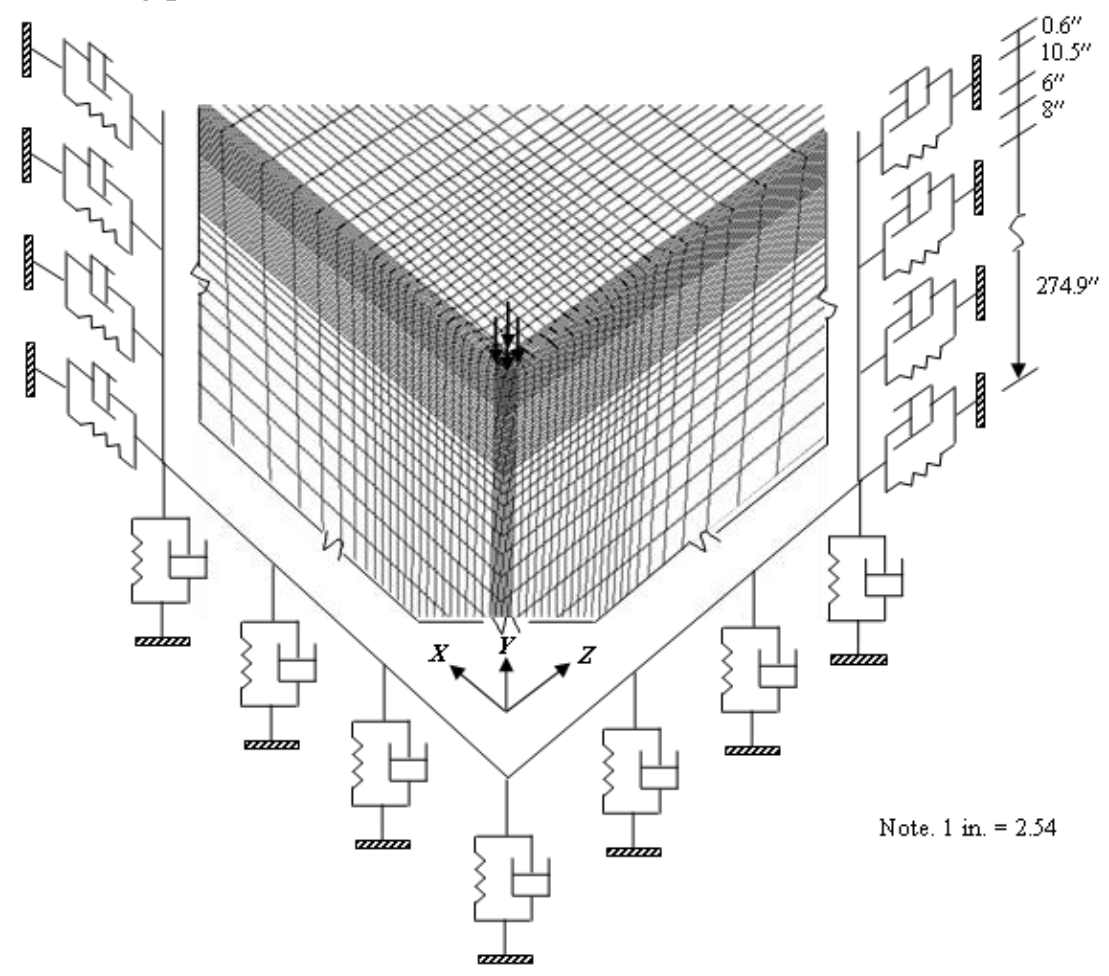

Figure 2. Mesh generation and boundary of FEM

The so-called spring-dashpots were used along the boundaries in the two mutually orthogonal directions considering the future application of this model under repeated load as shown in Figure 2 (Lysmer and Kuhlemeyer 1969, and Gazetas 1991). It is expected that stresses due to the repeated load will not be reflected back from the boundary due to the use of spring-dashpots. The coulomb friction law was used to model the contact along the layer interfaces. Based on the visual inspection from coring, most of the layer interfaces were in good condition except the interface between the first and second lifts of the AC layer. Therefore, only this interface was considered as partially-bonded whereas the rest of the layer interfaces were considered as fully-bonded. The friction coefficients required for this contact model were collected from the literature (Romanoschi and Metcalf 2001). The friction coefficient along partially-bonded interfaces in $\mathrm{AC}$ is 0.7 and that along the other interfaces is 1.0. 


\section{Properties of Material}

\section{AC layer}

Field-compacted AC cores were collected using a 6 in. diameter core drill bit from the pavement section. Both vertical and horizontal cores were extracted from these cores using $3 \mathrm{in}$. diameter core drill bit in the laboratory. Figure 3(a) shows both of these vertical and horizontal cores. Height and diameter of the cores were $4.5 \mathrm{in}$. and $3 \mathrm{in}$. respectively. After the sample preparation, the dynamic modulus tests were conducted on both vertical and horizontal AC cores at different temperatures $\left(-10,4,21,37\right.$, and $\left.54{ }^{\circ} \mathrm{C}\right)$ and frequencies $(0.1,0.5,1,5,10$ and $25 \mathrm{~Hz})$ according to the AASHTO TP 62-07 (2007). A dynamic master curve was generated from these test results using the Time-Temperature Superposition (TTSP). Later, dynamic modulus master curves for both of the vertical and horizontal AC cores were converted to relaxation modulus curves according to the method of conversion as proposed by Park and Schapery (1999). These vertical and horizontal relaxation modulus curved were used to fit the following Prony series equation based on the Generalized Maxwell Model as follows:

$$
\begin{aligned}
& E_{v}(t)=E_{0, v}\left[1-\sum_{i=1}^{m} e_{v, i}\left(1-e^{-t / \tau_{v, i}}\right)\right] \\
& E_{h}(t)=E_{0, h}\left[1-\sum_{i=1}^{m} e_{h, i}\left(1-e^{-t / \tau_{h, i}}\right)\right]
\end{aligned}
$$

where $E_{v}(t)=$ vertical relaxation modulus $(\mathrm{ksi}), E_{h}(t)=$ horizontal relaxation modulus $(\mathrm{ksi}), E_{0, v}=$ vertical instantaneous modulus (ksi), $E_{0, h}=$ horizontal instantaneous modulus (ksi), $e_{v, i}=$ weight factor of $i$-th spring-dashpot for vertical modulus, $e_{h, i}=$ weight factor of $i$-th spring-dashpot for horizontal modulus, $\tau_{v, l}=$ relaxation time for vertical modulus, $\tau_{h, i}=$ relaxation time for horizontal modulus, and $m=$ total number of spring-dashpots. These parameters are summarized in Table 1. Vertical and horizontal instantaneous moduli are 7037 and 3795 ksi respectively.

\section{Table 1. Prony series coefficients of vertical and horizontal AC test specimen}

\begin{tabular}{|c|c|c|c|c|}
\hline$i$ & $e_{v, i}$ & $\tau_{v, i}$ & $e_{h, i}$ & $\tau_{h, i}$ \\
\hline 1 & 0.277 & $1.04 \mathrm{E}-05$ & 0.292 & 0.001 \\
\hline 2 & 0.2 & 0.018 & 0.2 & 0.056 \\
\hline 3 & 0.15 & 0.0011 & 0.15 & 15.64 \\
\hline 4 & 0.13 & 0.00019 & 0.19 & 0.0001 \\
\hline 5 & 0.13 & 0.68 & 0.1 & 0.61 \\
\hline 6 & 0.09 & 22.99 & 0.054 & 0.02 \\
\hline
\end{tabular}

These coefficients are also assumed to be same for the shear modulus except the instantaneous shear modulus. The equations of the instantaneous shear modulus are as follows:

$G_{v h}(t)=n^{\prime} \times E_{v}(t)$

$G_{h h}(t)=\frac{E_{h}(t)}{2\left(1+v_{h h}\right)}$

where $G_{v h}(t)=$ shear modulus in vertical plane (ksi), $n^{\prime}=$ factor for shear modulus $(=0.38)(18)$, $G_{h h}(t)=$ shear modulus in horizontal plane (ksi), and $v_{h h}=$ Poisson's ratio in horizontal plane $(=0.3)$. 
Temperature dependency of the AC is incorporated using the following relationship which was developed from a regression analysis based on backcalculated AC modulus from the Falling Weight Deflectometer (FWD) tests on the instrumented pavement section at different temperatures:

$\frac{E_{21^{\circ} \mathrm{C}}}{E_{T}}=e^{-0.0342(T-21)}$

where $E_{21^{\circ} \mathrm{C}}=$ modulus of the $\mathrm{AC}$ at $21{ }^{\circ} \mathrm{C}, E_{T}=$ modulus of the $\mathrm{AC}$ at $T^{\circ} \mathrm{C}$, and $T=$ temperature of the $\mathrm{AC}$ at a specific depth $\left({ }^{\circ} \mathrm{C}\right)$. A FORTRAN subroutine is developed to implement the temperature dependent and cross-anisotropic viscoelastic model of the AC layer. This subroutine is integrated to the dynamic FEM in ABAQUS using the User Defined Material (UMAT) interface.
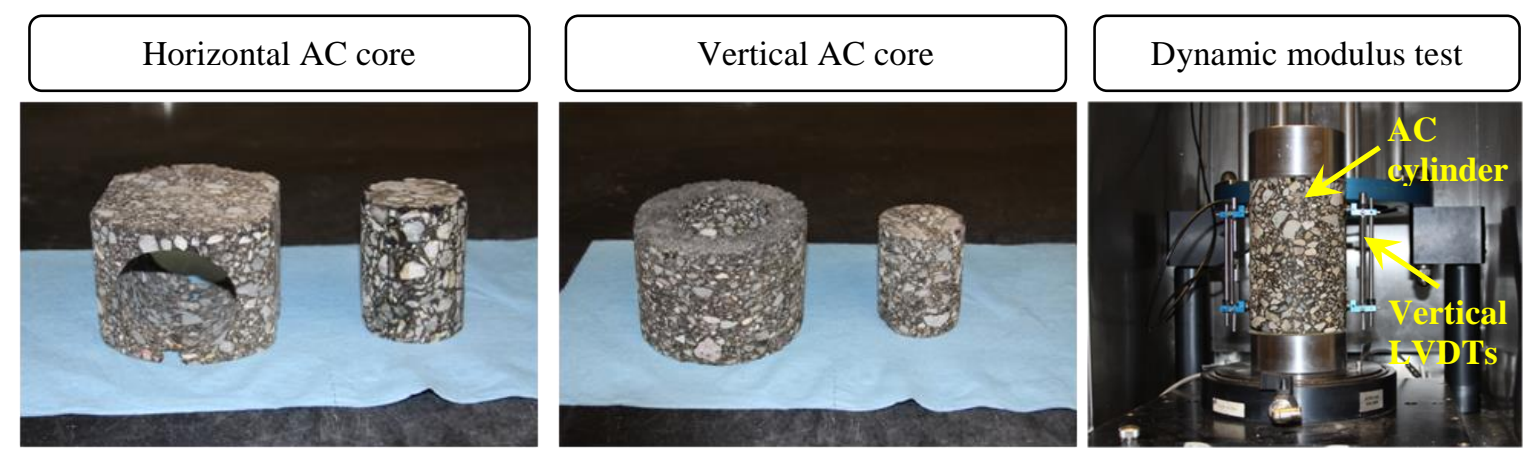

(a) AC sample and dynamic modulus test modulus
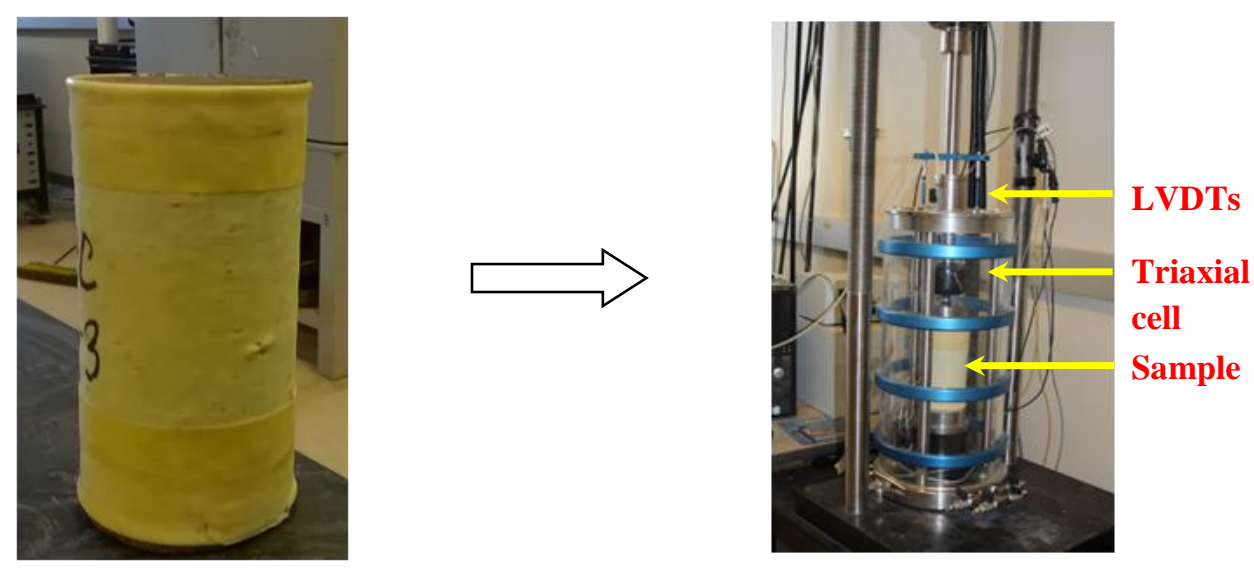

(b) Unbound layer sample and resilient modulus test

\section{Figure 3. Laboratory tests for development of material models}

\section{Unbound Layer}

The two unbound layers, i.e., base and subbase, are considered nonlinear elastic and stress-dependent materials in the dynamic FEM of the pavement section. Laboratory resilient modulus tests were conducted on both of these layer materials to determine the stress-dependencies according to the AASHTO T307-99 (2003). Figure 3(b) shows that a cylinder test specimen of unbound material with 4 in. diameter and 8 in. height was prepared for a resilient modulus test. External LVDTs were used to measure axial displacements under repeated load. Resilient modulus was determined for a number stress sequences according to the test protocol. The generalized model as adopted in the newly developed Mechanistic Empirical Pavement 
Design Guide (MEPDG) was used in this study to incorporate base and subbase nonlinearity to the following model:

$$
M_{r}=k_{1}\left(\frac{\theta}{p_{a}}\right)^{k_{2}}\left(\frac{\tau_{o c t}}{p_{a}}+1\right)^{k_{3}}
$$

where $\theta=$ bulk stress (psi), $\tau_{o c t}=$ octahedral shear stress (psi), $p_{a}=$ atmospheric pressure (= $\left.14.65 \mathrm{psi}\right)$, and $k_{1}, k_{2}, k_{3}=$ regression coefficients that need to be determined from laboratory resilient modulus test. Values of these coefficients are $12384,0.15$, and 0.75 respectively for base whereas $3285,0.17$, and -0.27 respectively for subbase. Eq. (6) is also implemented in dynamic FEM using UMAT interface in ABAQUS. The subgrade is assumed as linear elastic since the stress variation under a wheel is small and modulus of elasticity is $25 \mathrm{ksi}$ as backcalculated from the FWD test. Densities of the AC, base, subbase, and subgrade are $145,135,120$, and 110 pcf respectively. Poisson's ratios of the AC, base, subbase, and subgrade are $0.35,0.4,0.4$, and 0.45 respectively.

\section{Load and Temperature}

In this study, the FEM is simulated under a Falling Weight Deflectometer (FWD) test load. During a FWD test, an impulse type load of 9 kip is applied over a circular area with radius of 6 in to produce 79.6 psi. This load varies following a haversine pattern over a loading duration of 30 milliseconds (Ahmed et al. 2010). Two different temperature cases are considered: average temperature constant over the depth of the $\mathrm{AC}$ layer and differential temperature in AC layer. Details of these two temperature distributions are discussed below:

In case of constant temperature distribution in AC layer, average of the surface and bottom temperatures are determined. The measured surface and bottom temperatures are 9.9 and $5.4{ }^{\circ} \mathrm{C}$ respectively. The resulting average temperature is $7.65^{\circ} \mathrm{C}$. In case of differential temperature, temperature is assumed to vary linearly over the depth of the AC layer. Based on this assumption, the equation for the temperature variations is as follows:

$T_{z}=T_{\text {surface }}-\frac{\left(T_{\text {surafce }}-T_{\text {bottom }}\right) \times z}{D}$

where $T_{z}=$ temperature of the $\mathrm{AC}$ at depth, $z\left({ }^{0} \mathrm{C}\right), T_{\text {surface }}=$ surface temperature $\left({ }^{0} \mathrm{C}\right), T_{\text {bottom }}=$ temperature at bottom of the AC layer $\left({ }^{0} \mathrm{C}\right)$, and $D=$ thickness of the AC layer (in.). Temperatures at different depths are: $T_{z}=T_{\text {surface }}=9.9{ }^{\circ} \mathrm{C}$ where $0 \leq z \leq 0.6$ and $T_{\text {bottom }}=5.4{ }^{\circ} \mathrm{C}$ which was recorded by the temperature probes in January.

\section{Results and Discussion}

\section{FEM Validation}

The developed dynamic FEM is simulated under the FWD test load of 9kip. Simulated deflections are compared with those measured by deflection sensors located at different radial distances. Figure 4(a) shows the time-deflection histories as measured from field test and simulated by the FEM at the center of the loading area. Peak of the field time-deflection history is 5 mil, i.e., 0.005 in., and it is slightly smaller than the peak in the simulated time-deflection history. The qualitative shapes of both of these time-deflection histories are similar. Peak deflections of time-deflection histories from five deflection sensors located at 0 , $8,12,18$, and 24 in. from the center of the loading area are plotted in Figure 4(b). Plot of deflection variation over radial distances is known as deflection basin. Deflection basins from field measurement and FEM simulation are compared. Deflection differences in near sensors are very small and it increases toward the 
far sensors. The Root Mean Squared Error (RMSE) of these deflection basins is determined. The RMSE $(\%)$ is 7.1 which is below $10 \%$. Based on the overall deflection comparison, it can be claimed that the FEM is validated and it is used for further simulation in the next step.

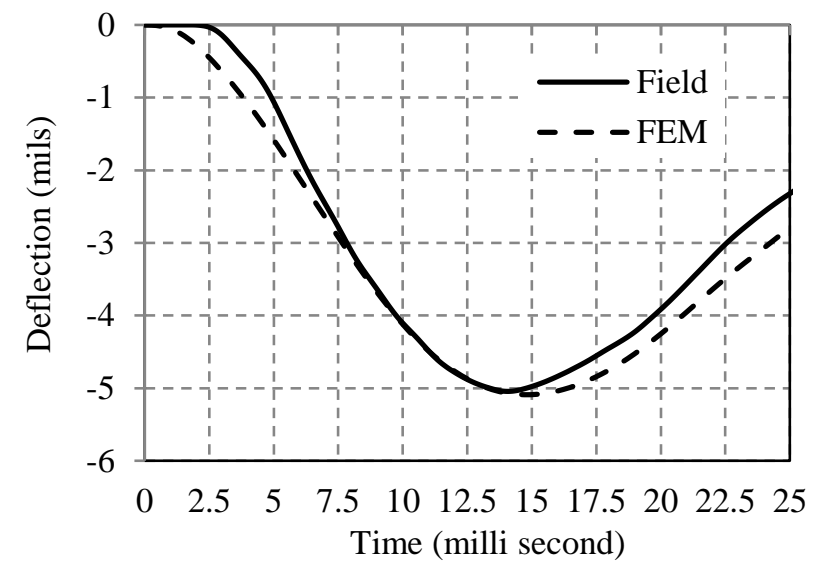

(a) Time-deflection history

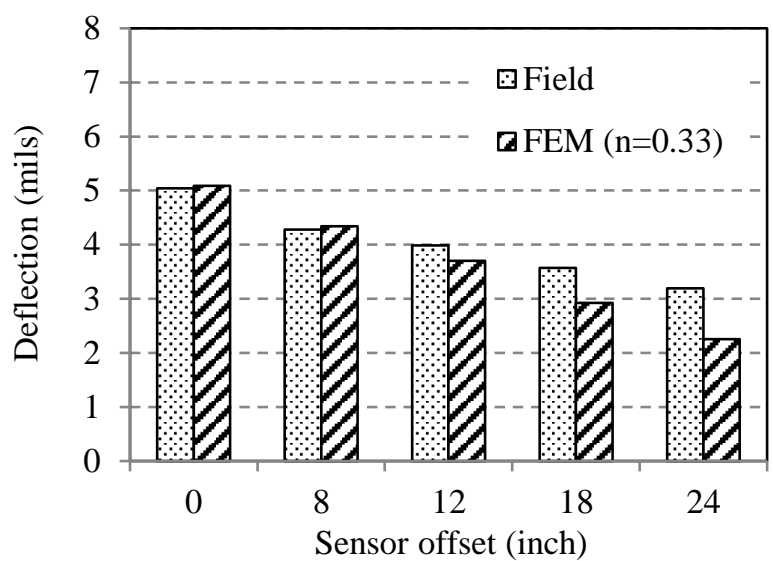

(b) Deflection basin

Figure 4. Comparison of deflections between field and FEM

\section{Pavement Deflections}

The validated dynamic FEM is simulated incorporating both average and differential depth-temperature profiles. Simulated time-deflection histories on top of the AC and subgrade considering average depthtemperature profile are plotted in Figure 5(a). It is observed that the peak of AC-top time-deflection history is greater than the peak subgrade-top time deflection history which is expected. It is also observed that the time lag between these two peak deflections is about 3 milliseconds. This time lag is due to the time needed for the stress wave travel from the AC-top to the subgrade-top. It indicates that the peak deflections in every layer are not observed in the same time. Times of attaining peak deflections at different depths depend on velocity of stress wave. This velocity is dependent on material properties, i.e., $v=\sqrt{E / \rho}$ where $E=$ modulus of elasticity and $\rho=$ density. It indicates that the temperature variation affects this deflection time lag based on temperature dependency of modulus of elasticity and density.

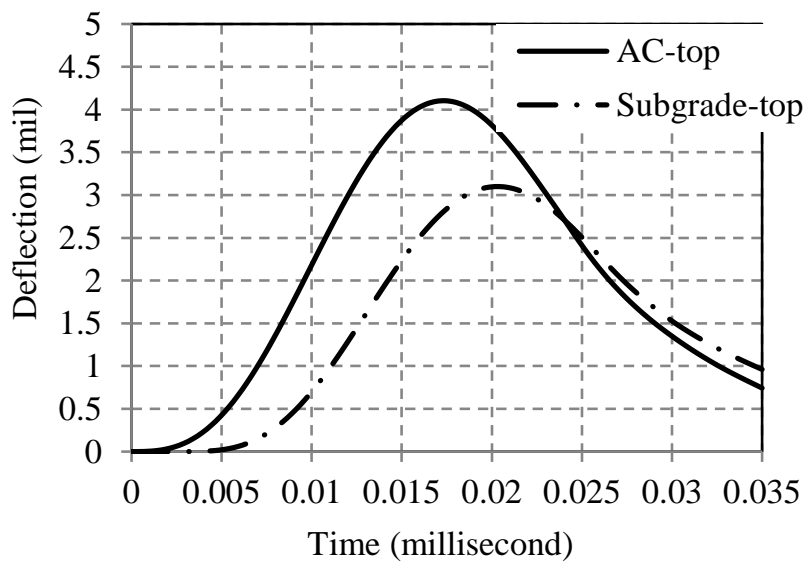

(a) Time-deflection history

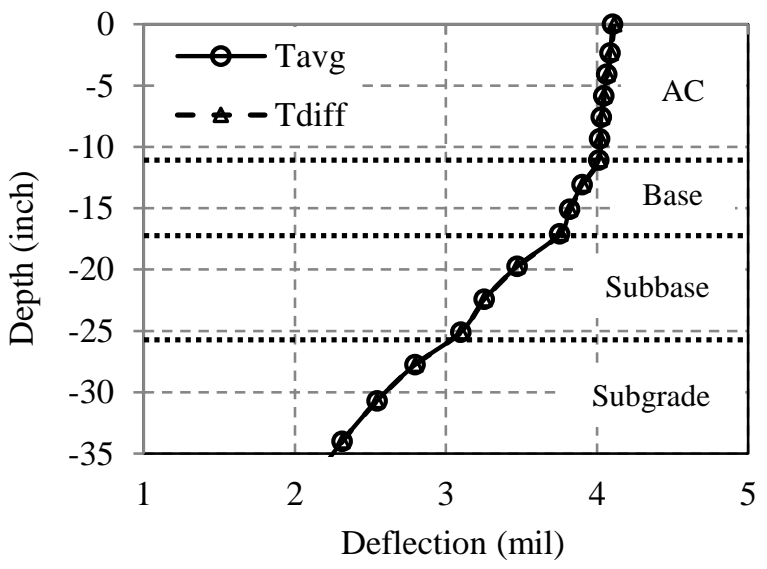

(b) Deflection profile

Figure 5. Effect of temperature distribution on deflections 
Now, peak deflections are recorded from the time-deflection histories at different depths at the center of the loading area. These peak deflections are plotted against pavement depths considering both average and differential temperature distribution in Figure 5(b). It is observed that deflection decreases with depth and rate of deflection decrease is high in unbound layers, i.e., base, subbase, and subgrade, compared to the AC layer. Difference in deflection due to the two different temperature distribution cases is very small. The RMSE (\%) of the deflections at these two temperature distributions is 0.11 . Figure 6 shows the variation of $\mathrm{AC}$ modulus due to depth-temperature profile. AC modulus is low at high pavement surface temperature whereas this modulus is high at the bottom of the AC layer. The RMSE (\%) of AC modulus based on these two temperature distributions is 5.11. However, this magnitude of RMSE has not high effect on pavement deflections. Based on the observation, deflection over pavement depth is not affected by the average and differential temperature distribution in the AC layer.

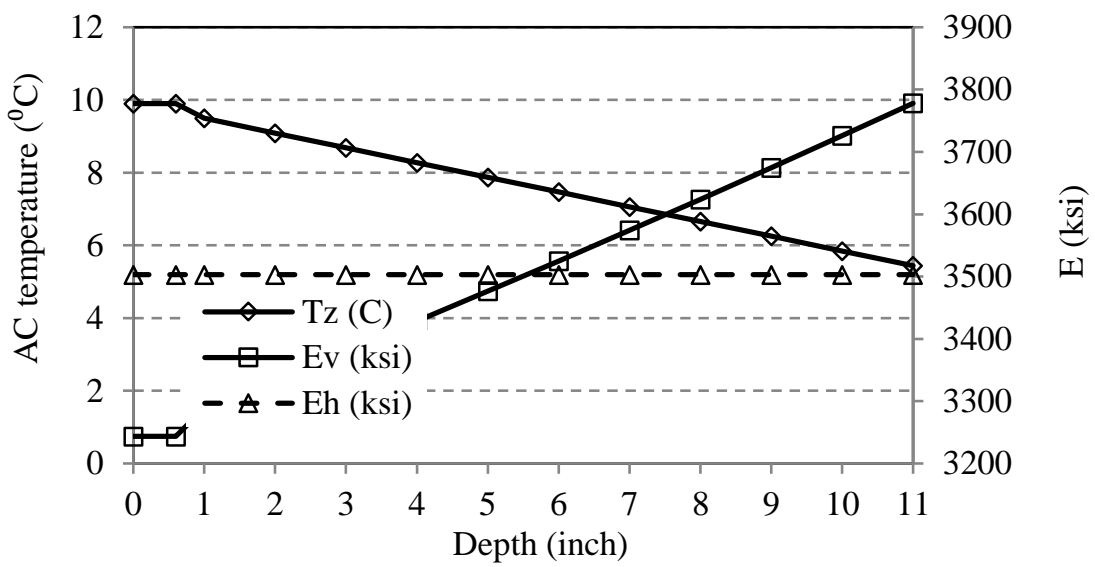

Figure 6. Depth-temperature distribution and modulus variation

\section{Stress and Strain}

In the earlier step, it is observed that the rate of deflection decrease is high in unbound layers. The depthvertical strain profiles are plotted for both of the temperature distributions in Figure 7(a). It is observed that the vertical strain variation in the AC is the smallest and there is sudden increase at the AC-base layer interface which is due to the change in material properties. Strain gradually decreases with and again, sudden increase in strain at base-subbase layer interface is observed which is due to the same reason. Vertical strains in both subbase and subgrade decreases gradually with depth. In addition, trends of these strain variations in these layers are close. Now, referring to Figure 5(b), deflection variations in subbase and subgrade are greater than the two overlain layers which are actually the results of the strain variation in Figure 7(a). Finally, two different types of temperature distribution have very little effect on strain which is evident since the RMSE (\%) of strains over pavement depths is equal to 1.1 .

Figure 7(b) shows the depth-vertical stress profiles due to two different temperature profiles in the AC layer. It is observed that the stress decreases with depth and rate of decrease is high in the AC layer. Most of the stress, i.e., about 60 psi, is distributed in this layer below this layer, stress drops below 20 psi. In strain variation in Figure 7(a), vertical strains are high in unbound layers under stresses below 20 psi. This reason is that the unbound layer moduli is very small compared to the AC relaxation modulus. Stress variations at two different depth-temperature profiles are also very close and the RMSE (\%) is 1.25 . In summary, both of the vertical stress and strain are not sensitive to the temperature variation.

Tensile strain at the bottom of the AC layer is also determined at two different temperature distribution cases. In case of differential temperature distribution, tensile strain is 29.6 microstrain whereas this strain is 29.8 microstrain in case of average temperature distribution. These values are very close which indicates that the tensile in AC layer is not affected by the differential temperature distribution over the AC depth. 


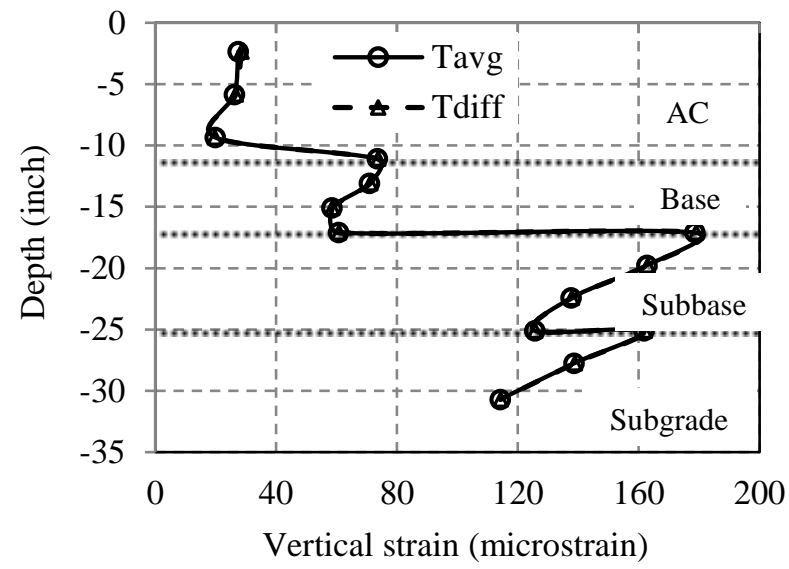

(a) Vertical strain profile

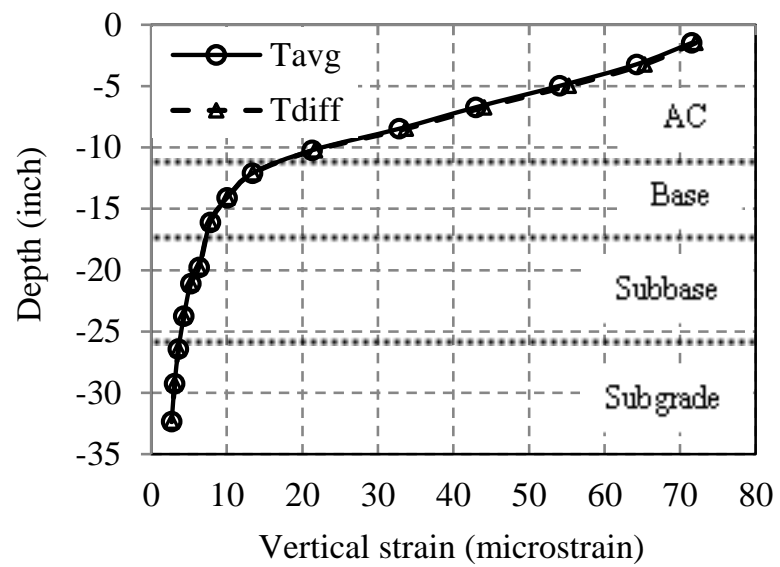

(b) Vertical stress profile

Figure 7. Effect of temperature distribution on vertical stress and strain

\section{CONCLUSION}

A dynamic FEM is developed to investigate the effect of differential temperature distribution in an AC layer on pavement responses in presence of AC cross-anisotropy and unbound layer stress-dependency. Based on the FEM simulations, the observations are as follows:

- AC modulus varies over depth of an AC layer due to differential temperature at different depths.

- Pavement deflections at different depths are almost same in case of both differential temperature and average constant temperature over the depth of an AC layer.

- Vertical stress and strain are the same over the pavement depth in cases of two different temperature distributions. This observation is also common for tensile strain at the bottom of the AC layer.

Based on the observation, it is evident that there is no significant difference between the pavement responses, such as deflections, stress, and strain, due to incorporation of average temperature and differential depth-temperature profiles even after the temperature dependency of the AC modulus. Therefore, accuracy of pavement analysis and thereby, distress calculation will not compromised due to consideration of average temperature over the depth of an AC layer.

\section{REFERENCES}

AASHTO TP 62-07. (2007). "Standard Method of Test for Determining Dynamic Modulus of Hot Mix Asphalt Concrete Mixtures." AASHTO Provisional Standards, Washington, D. C.

AASHTO. (2003). "Standard Method of Test for Determining the Resilient Modulus of Soils and Aggregate Materials." AASHTO T307-99, Washington, D.C.

Ahmed, M. U. (2010). "Evaluation of FWD Software and Deflection Basin for Airport Pavements." M.Sc Thesis, University of New Mexico.

Ahmed, M. U., Rahman, A., Islam, M. R., and Tarefder, R. A. (2015). "Combined Effect of Asphalt Concrete Cross-Anisotropy and Temperature Variation on Pavement Stress-Strain under Dynamic Loading." Construction and Building Material, Journal of Elsevier, 93, 685-694.

Diefenderfer, B. K. (2002). "Moisture Content Determination and Temperature Profile Modeling of Flexible Pavement Structures." PhD Dissertation, Civil and Environmental Engineering, Virginia Polytechnic Institute and State University. 
Duncan, J. M., Monismith, C. L., and Wilson, E. L. (1968). "Finite Element Analyses of Pavements." Highway Res. Rec.: J. of Highway Res. Board, 228, 18-23.

Gazetas, G. (1991). "Formulas and Charts for Impedances of Surface and Embedded Foundations." In Journal of Geotechnical Engineering, ASCE, Vol. 117, pp. 1363-1381.

Herb, W., Marasteanu, M., and Stefan, H. G. (2006). "Simulation and Characterization of Asphalt Pavement Temperatures.” Project Report NO. 480, Minnesota Department of Transportation (MNDOT).

Lysmer, J., and Kuhlemeyer, R. L. (1969). "Finite Element Model for Infinite Media." In Journal of Engineering Mechanics, ASCE, Vol. 95, pp. 859-877.

Park. S. W., and R. A. Schapery. (1999). "Methods of Interconvertion between Linear Viscoelastic Material Functions. Part I- A Numerical Method Based on Prony Series.” International Journal of Solids and Structures, Vol. 36, pp. 1653-1675.

Petyt, M. (1990). "Introduction to Finite Element Vibration Analysis." $1^{\text {st }}$ Edition. Cambridge University Press, Cambridge, UK.

Romanoschi, S. A., and Metcalf, J. B. (2001). "Characterization of Asphalt Concrete Layer Interfaces.” In Transportation Research Record: Journal of Transportation Research Board, 1778, 2001, pp. 132-139.

Tarefder, R. A., Ahmed, M. U., and Rahman, A. (2016). "Effects of Cross-Anisotropy and StressDependency on Pavement Layers on Pavement Responses under Dynamic Truck Loading." Journal of Rock Mechanics and Geotechnical Engineering, Elsevier. (in press)

Wang, H., and Al-Qadi, I. L. (2013). "Importance of Nonlinear Anisotropic Modeling of Granular Base for Predicting Maximum Viscoelastic Pavement Responses Under Moving Vehicular Loading." Journal of Engineering Mechanics, Vol. 139, No. 29, pp. 29-38. 